\title{
Zur Lebenssituation und Zukunftsperspektive von eritreischen Geflüchteten: Geflohen nach Israel - deportiert nach Ruanda/Uganda - gestrandet in Kampala
}

\author{
Gabriele Rosenthal, Lukas Hofmann
}

\section{Einleitung}

In unserem Beitrag gehen wir der Frage nach, wie die Lebenssituationen und $\mathrm{Zu}$ kunftsperspektiven von Menschen aussehen, die aus von massiver kollektiver Gewalt geprägten Kontexten geflohen sind, etliche Jahre in einem anderen Land gelebt und gearbeitet haben, dann in ein weiteres Land deportiert wurden, in dem sie heute in fast auswegloser Situation leben. Wir konzentrieren uns dabei auf die Gruppierung von Eritreern, die nach Israel geflohen waren, die meisten zwischen 2010 und 2012, ${ }^{1}$ dort mehrere Jahre lebten, Arbeit fanden, teilweise heirateten und Kinder

\footnotetext{
${ }^{1}$ Bis Ende 2012 flohen 37.347 Eritreer*innen nach Israel (vgl. https://esa.un.org/MigGMGProfiles/indicators/files/Eritrea.pdf <02.09.2020>); danach wurde die Überwindung des in 2012 errichteten Grenzzaunes zwischen Agypten und Israel, dessen Bau 2014 abgeschlossen war, immer schwieriger und die Route über den Sinai immer lebensgefährlicher. Im Jahr 2017 lebten nur noch 22.000 Eritreer*innen in Israel (vgl. https://www.unhcr.org/5b27be547.pdf, S. $15<09.05 .2019>$ ). Es sei an dieser Stelle erwähnt, dass wir im Folgenden immer wieder auf Berichte von NGOs und aus den Massenmedien hinweisen. Auch sind viele Informationen bisher nicht in Fachpublikationen erschienen bzw. 
bekamen sowie schließlich, unter massivem Druck zur Ausreise in ein sogenanntes ,sicheres' afrikanisches Land genötigt, dieser Auflage zustimmten. Der Druck erfolgte vor allem durch die Internierung im 2013 eröffneten Lager Holot in der Wüste Negev, dem damit verbundenen Verlust des Arbeitsplatzes und durch Verhängung von hohen Haftstrafen für geringfügige Delikte (vgl. Birger/Shoham/Bolzman 2018). Die von uns zwischen 2015 und 2018 in Kampala, der Hauptstadt Ugandas, interviewten, ${ }^{2}$ aus Israel ausgewiesenen Männer (Frauen waren bisher von diesem massiven Druck zur Ausreise noch nicht betroffen) landeten entweder in Ruanda oder in Uganda. Meist wurden sie erst auf dem Flug dorthin über den Zielort informiert. Diejenigen, die in Ruanda ankamen, erhielten nur ein kurzfristig geltendes Touristenvisum und sahen sich deshalb gezwungen, das Land nach wenigen Wochen wieder zu verlassen. Sie überquerten dann - mit Hilfe von sich andienenden Schleppern - „illegal“ die Grenze nach Uganda. In Kampala fanden sie eine recht große Community von aus Eritrea über den Sudan oder per Flugzeug über Äthiopien Geflüchteten vor. ${ }^{3}$ Im Unterschied zu den aus Israel faktisch Ausgewiesenen, die nur unter bestimmten Bedingungen Asyl beantragen können und damit in einer rechtlich ungeschützten Situation im Land leben, können die auf anderen Wegen Eingereisten, wenn sie über entsprechende finanzielle Mittel verfügen, ${ }^{4}$ in Uganda einen befristeten Asylstatus beantragen und immer wieder verlängern. Die aus Israel Ausgewiesenen können dagegen nur dann Asyl beantragen, wenn sie ihre Zeit in Israel verschweigen, da Ruanda und Uganda die Existenz jeglicher Abkommen mit Israel über die Aufnahme von aus Israel Ausgewiesenen abstreiten. ${ }^{5}$

Gemeinsam ist fast allen, dass sie auf eine völlig unsichere Zukunft blicken und sich nach Möglichkeiten der Migration in ein anderes Land, auch dem Wiederzusammenkommen mit Familienangehörigen umschauen, nicht allzu selten und immer wieder in Gefahr sind, ihre Handlungsmacht mehr oder weniger zu verlieren, und in völliger Depression und Apathie zu versinken drohen. Resultierende Ohnmachtsgefühle werden unseren Beobachtungen nach teilweise durch Drogenmissbrauch,

beziehen sich diese auf ähnliche Quellen. Wir überprüften die Angaben allerdings jeweils anhand von mehreren Quellen, die wir aus Platzgründen nicht alle angeben können.

2 Alle Interviews wurden mit einer narrativen Gesprächsführung durchgeführt (vgl. Rosenthal 2015: Kap. 5.).

3 Im April 2019 lebten 10.391 gemeldete Eritreer*innen in Kampala (https://data2.unhcr.org/en/documents/details/69444 <18.07.2019>). Ein Drittel der Bevölkerung Eritreas lebt in der Diaspora!

${ }^{4}$ Neben den Beträgen, die sie auf der ugandischen Botschaft für einen Antrag bezahlen müssen, setzt der Antrag auch die Ausstellung von Papieren auf der eritreischen Botschaft voraus. Diese verlangt einen Beitrag für die Ausreise aus Eritrea sowie rückwirkend 2\% ihres Einkommens, die so genannte „recovery tax“, die es dauerhaft zu zahlen gilt. Als Druckmittel dienen die Verwandten in Eritrea (https://www.dsp-groep.eu/wp-content/uploads/The-2-Tax-for-Eritreans-in-the-diaspora_30-august-1.pdf, S. $10<09.05 .2019>$ ).

${ }_{5}^{5}$ Während wir diesen Artikel schrieben, erschien am 08.07.2019 im Daily Monitor, der wichtigsten englischsprachigen Oppositionszeitung Ugandas, ein Artikel von Kakkooza und Mutaizibwa, in dem über den „secret deal that violates international law obligations“ zwischen Israel und Uganda berichtet wird (https://www.monitor.co.ug/SpecialReports/Stateless-Eritreans-secretly-moved-Uganda/ 688342-5186934-o26emx/index.html <02.09.2020>). 
insbesondere mit Konsum von Khat in Verbindung mit Tabak und starkem Kaffee (beide verstärken die Wirkung von Khat), bekämpft. Die dank der Drogen zunächst erlebte Euphorie, einhergehend mit Gefühlen von Stärke oder Unbesiegbarkeit, verfestigt im Verlauf wiederum die depressive Grundstimmung. Etwas Hoffnung für jene, die in Äthiopien ein Leben in sozialer und ökonomischer Sicherheit erwarten können, gibt mittlerweile der im Juli 2018 zwischen Äthiopien und Eritrea unterzeichnete Friedens- und Freundschaftsvertrag und die damit verbundene Öffnung der Grenzen. ${ }^{6}$

Die Verläufe der aus Eritrea geflohenen Menschen verdeutlichen, wie wenig ihnen eine binäre Unterscheidung von Flucht und Migration gerecht werden würde, wie es z.B. von Arne Worm (2019) anhand von aus Syrien nach Europa geflohenen Menschen überzeugend gezeigt wurde. Wie bei vielen Menschen, die aus stark von alltäglicher sowohl kollektiver als auch familialer Gewalt geprägten Kontexten manchmal zunächst nur aus einer in eine andere Region ihres Heimatlandes geflohen sind, handelt es sich um Verläufe, in denen sich Phasen von starkem Erleiden bzw. von Machtlosigkeit oft mit Phasen einer gewissen Handlungsmacht abwechseln. Bei den eritreischen Geflüchteten in Uganda beginnt in der Situation des Gestrandetseins wieder eine Phase, die stark von eher passivem Erleiden geprägt ist und die zur Reaktivierung von zurückliegenden traumatisierenden Erlebnissen führen kann. Bei unseren Analysen der Lebenswege dieser Menschen zeigten sich immer wieder Verläufe, bei denen nicht nur einzelne traumatisierende Situationen während der Flucht oder Migration erlebt wurden, sondern bei denen wir von einer sequentiellen Traumatisierung im Sinne Hans Keilsons (1979) oder David Beckers (2007) auszugehen haben. Keilson, der darunter einen langfristigen Prozess und ein Ineinandergreifen von mehreren traumatischen Sequenzen versteht, wies darauf hin, dass eine schwierige Zeit nach der erlittenen Gewalt zur Fortsetzung und Verstärkung der Traumatisierung führen kann. Die von uns Interviewten erlebten traumatisierende Situationen in ihrer Heimat, meist im Kontext ihres unbefristeten „Nationaldienstes“, 7 auf der Flucht, bei der Überquerung von regionalen und nationalen Grenzen. Viele Frauen, aber auch Männer wurden auf der Flucht oder schon im Militär vergewaltigt. Auf dem Sinai wurden etliche von ihnen entführt und gefoltert, um Lösegeld von ihren Verwandten zu erpressen. Einige wurden zu Zeugen von Organentnahmen und dem darauffolgenden Tod von Mitgefangenen. ${ }^{8}$ Auch in Israel, wo sie offiziell

\footnotetext{
${ }^{6}$ Zur Situation an der Grenze und zu zunehmenden Asylanträgen von Eritreern in Äthiopien siehe: https://www.nrc.no/news/2018/october/ethiopia---eritrea/ <18.07.2019>.

7 Seit 1995 besteht der „Nationaldienst“ für Männer und Frauen vom 18. bis offiziell zum 47. Lebensjahr, in manchen Fällen sogar bis zum 59. Lebensjahr (vgl. Bartolucci 2017). Die Angaben zur Länge des Dienstes unterscheiden sich teilweise in der Literatur - vielleicht, weil die Angaben der eritreischen Regierung nicht unabhängig überprüft werden können (vgl. https://www.fluechtlingshilfe.ch/filead$\mathrm{min} /$ user_upload/Publikationen/Herkunftslaenderberichte/Afrika/Eritrea/170630-eri-nationaldienst-de.pdf $<02.09 .2020>$ ).

${ }^{8}$ Von diesen traumatisierenden Erlebnissen berichten die Interviewten teilweise sehr detailliert. Es gibt dazu zahlreiche Veröffentlichungen. Hier möchten wir auf die empirische Untersuchung von Mekonnen und Estefanos (2012) verweisen, die 2011 mehr als 100 narrative Telefoninterviews mit noch auf dem Sinai gefangenen Eritreern führten sowie Interviews auswerteten, die im Auftrag von
} 
als „infiltrators“ bezeichnet und so stigmatisiert werden, erlebten sie demütigende Situationen, sowohl im Alltagsleben als auch und vor allem bei der Internierung in israelischen Gefängnissen und Lagern. All diese Erlebnisse können in der prekären Situation in Uganda oftmals in der Erinnerung reaktiviert werden. Die Angst vor Diskriminierung und Gewalt, vor dem eritreischen Geheimdienst, den mit ihm kooperierenden anderen Geheimdiensten und vor allem vor einer Verfolgung der noch in Eritrea lebenden Verwandten bestimmt ihren Lebensalltag. Die für sie im Raum stehende Gefahr einer Verschleppung zurück nach Eritrea reaktiviert insbesondere die Erinnerungen an im eritreischen Militär erlittene Traumata. Die Flucht aus Eritrea war auch meist durch die konkreten Umstände während des Militärdienstes, der seit 2002 in den mehr oder weniger unbefristeten „Nationaldienst“ übergeht, ausgelöst worden. Beim „Nationaldienst“ handelt es sich um eine Form der Zwangsarbeit in unterschiedlichen Arbeitsbereichen mit geringer Bezahlung (ca. 10-40 US-Dollar monatlich), die zum Wiederaufbau des Landes dienen soll. Damit werden den Familien ihre Arbeits- und Verdienstmöglichkeiten fast völlig zerstört (vgl. Kibreab 2017a). Die ausgesprochen schwierigen und gefährlichen Lebensbedingungen beim Militär und in den verschiedenen Einsätzen gingen meist noch mit Inhaftierungen und Folter wegen geringfügigster Vorkommnisse und für die Frauen oft mit dem Erleiden von sexualisierter Gewalt einher (vgl. Kibreab 2017b). ${ }^{9}$

Wir nehmen an, dass unsere empirischen Befunde zu Fluchtmigrationen und zur gegenwärtigen Lebenssituation von Eritreern in Uganda nicht nur auf diese Gruppierung zutreffen. Es sind Menschen, die in verschiedenen Ländern staatlicher Willkür und Gewalt ausgesetzt waren und sind, hinter deren Rücken und generell recht verborgen vor der internationalen Öffentlichkeit, teilweise auch massiv geleugnet, verschiedene Staaten ihre Abkommen schließen, die die Geflüchteten zu tendenziell passiven und zumeist hilflosen Objekten der Interessen der Regierungen und anderer mächtiger politischer Kräfte machen (vgl. Hirt 2016; Hirt/Mohammad 2018). Neben den Abkommen, die Israel mit afrikanischen Ländern hinsichtlich der Ausreise afrikanischer Asylsuchender aus Israel geschlossen hat, sind die Geflüchteten auch von den Vereinbarungen der EU zur Absicherung der europäischen Außengrenzen und zur Verhinderung der so genannten irregulären Migration und Bekämpfung „krimineller Netzwerke“ innerhalb des afrikanischen Kontinents betroffen. Die Grenzen innerhalb Afrikas werden vor allem seit November 2014 im Zusammenhang mit dem Khartum-Prozess deutlich undurchlässiger, da die EU verstärkt mit Abkommen zum Beispiel mit dem Sudan, aber auch mit Eritrea versucht, bereits weit außerhalb der EU-Grenzen so genannte irreguläre Migrant*innen abzuwehren.

„zivilgesellschaftlich“ oder humanitär aktiven NGOs in Israel geführt wurden. Zu Folter, Entnahme von Organen und Vergewaltigung von Frauen auf dem Sinai siehe außerdem die auf Interviews beruhende Untersuchung von Tesfagiorgis (2013).

${ }^{9}$ Zur sexualisierten Gewalt im Militär siehe auch den ausführlichen Bericht der Schweizerischen Flüchtlingshilfe von 2018, der etliche Quellenangaben enthält: https://www.ecoi.net/en/file/local/1425110/1788_1519377159_1302.pdf <02.09.2020>. 
Amnesty International befürchtet, dass in diesem Rahmen auch die Schließung der Grenzen Ugandas droht. ${ }^{10}$

Ebenfalls trifft unser empirischer Befund des Gestrandetseins in einer aussichtslosen Situation, nach vielen leidvollen Jahren der Fluchtmigration, die zeitweise und vorübergehend allerdings auch mit gewisser, erheblicher Handlungsmacht im Sinne der Umsetzung des eigenen Migrationsprojektes verbunden sind, auch auf andere Gruppierungen von Geflüchteten zu. Im Fall der von uns in Kampala interviewten Eritreer*innen sind die Möglichkeiten zu weiteren Migrationen sehr eingeschränkt, und sie sind in der Gefahr, ihre Handlungsfähigkeit und Kompetenzen, die sie im Laufe der Fluchtmigration erworben haben, zunehmend wieder zu verlieren. Selbst jene von uns interviewten Menschen, die sich in dieser hoffnungslosen Situation noch nicht völlig aufgegeben haben und immer wieder mit viel Energie versuchen, Wege für eine Migration in ein für sie ,sicheres' Land zu finden - wie der von uns später vorgestellte Fall von Robel ${ }^{11}$ zeigt -, blicken auf eine ausgesprochen unsichere Zukunft. Bevor wir den Fall von Robel sowie den von Abraham, eines ehemaligen Geheimdienstoffiziers, vorstellen, zunächst einige Informationen über unseren Forschungskontext und über unsere Schwierigkeiten beim Feldzugang, die maßgeblich mit der Angst der Befragten vor den Geheimdiensten verbunden war.

\section{Zum Forschungskontext: Erschwerter Feldzugang}

Während eines Aufenthaltes im Rahmen des DFG-Forschungsprojektes zu ExKindersoldat*innen in Norduganda (Bogner/Rosenthal 2018) ergab sich für G. Rosenthal die Möglichkeit, im Frühjahr 2015 und Frühjahr 2016 in Kampala sechs Eritreer zu interviewen, von denen vier aus Israel nach Ruanda abgeschoben worden waren, dann gezwungen waren, dieses Land ebenfalls zu verlassen, und in Uganda gestrandet waren. Drei von ihnen gelang einige Monate nach dem Interview der Weg über den Südsudan und Sudan nach Libyen sowie über das Mittelmeer nach Europa. Der vierte Mann, den wir Abraham nennen, kann diesen Weg nicht gehen, da dieser für ihn aufgrund seiner ehemaligen Funktion im eritreischen Geheimdienst und seinem Einsatz sowohl im Südsudan als auch in Darfur (im Westen der Republik Sudan) zu gefährlich ist. Die Reise in die Republik Sudan bedeutet speziell für aus Eritrea desertierte Offiziere und generell für eritreische Oppositionelle ein hohes Risiko

\footnotetext{
10 Vgl. http://amnesty-sudan.de/amnesty-wordpress/2017/02/17/europaeische-migrationspolitikder-khartoum-prozess / $<02.03 .2019>$.

${ }^{11}$ Alle Namen sind aus Gründen des Datenschutzes keine Klarnamen. Außerdem nennen wir zu ihrem Schutz manche Daten - wie z.B. den Herkunftsort, Namen von Gefängnissen, etc. - nicht. Auch Tom, der seit 2015 mit G. Rosenthal als Feldassistent zusammenarbeitet, Kontakte herstellte, Nachinterviews durchführte und vor allem die Übersetzungsarbeit übernahm, können wir nicht mit seinem Klarnamen nennen. Ihm sei an dieser Stelle für die ausgesprochen kompetente Mitarbeit und sein Engagement für unsere Untersuchung gedankt.
} 
(Hirt/Mohammad 2018: 117), vor allem da sie vom dort operierenden eritreischen Geheimdienst entführt und nach Eritrea deportiert werden könnten. ${ }^{12}$

Da sich diese Interviews als ausgesprochen hilfreich für das Verstehen der Fluchtmigration von Eritreer*innen erwiesen, die wir und unsere Kolleg*innen im Zusammenhang des DFG-Forschungsprojektes zur sozialen Konstruktion von Grenzgebieten $^{13}$ in Israel interviewt hatten, reisten wir im Februar/März 2018 zu einem weiteren Feldaufenthalt nach Kampala (Uganda). Damit fand diese Forschung im Kontext der für afrikanische Migrant*innen (vornehmlich aus dem Sudan und aus Eritrea) problematischen Entwicklung der Regierungspolitik in Israel statt, die sich im Frühjahr 2018 dramatisch zuspitzte. Die Entscheidung der israelischen Regierung im November 2017, alle alleinstehenden Männer der damals ca. 40.000 sich im Land befindenden Sudanes*innen und Eritreer*innen bis zum März 2018 in ein vermeintlich ,sicheres' afrikanisches Land auszuweisen, wurde verstärkt durchgesetzt. Der seit 2013 massiv herrschende Druck zu einer so genannten freiwilligen Abschiebung sollte zum Zwang werden. Bis zum April 2018 hatten sich bereits ca. 4.500 sudanesische und eritreische Geflüchtete - teilweise unter Androhung oder auch Vollzug von Gefängnisstrafen - drängen lassen, ihre Bereitschaft zur Ausreise in ein afrikanisches Drittland schriftlich zu erklären. Im Gegenzug erhielten sie 3.500 US-Dollar, Flugtickets, Reisedokumente und die Auszahlung des seit Mai 2017 einbehaltenen Anteils von 20 Prozent ihres Lohns. ${ }^{14}$ Die beabsichtigte Abschiebung aller Geflüchteten wurde von der israelischen Zivilgesellschaft stark kritisiert und im April 2018, d.h. einen Monat nach unserem Feldaufenthalt, wurde bekannt, dass die geplante Ausweisung aller Eritreer*innen und Sudanes*innen vom Obersten Gerichtshof in Israel gestoppt wurde. Dieser Rechtsspruch bietet jedoch keineswegs Sicherheit. So sollten z.B. im Januar 2019400 kongolesische Asylsuchende aus Israel ausgewiesen werden; dies wurde erneut gerichtlich untersagt. ${ }^{15}$

Im Frühjahr 2018 war unser Feldzugang in Kampala - ganz im Unterschied zu den Jahren zuvor - erheblich erschwert sowohl aufgrund massiver Ängste der Geflüchteten vor den israelischen, ugandischen und eritreischen Geheimdiensten als auch wegen einer aufgeladenen und ablehnenden Stimmung gegenüber Weißen bzw.

\footnotetext{
12 Die Regierung der Republik Sudan unter Umar al-Bashir stand immer wieder in enger Verbindung mit dem eritreischen Präsidenten Isayas Afewerki, und die Eritreer fürchten, dass sie aufgegriffen und nach Eritrea deportiert werden, wie es auch immer wieder geschieht (vgl. Hirt 2016: 3). Magnus Treiber (2013) und Kurt Beck konnten im März 2011 mit einer Gruppe eritreischer Offiziere in Khartum sprechen, die aus dem Militär desertiert waren und ihnen über ihre Sicherheitsvorkehrungen gegen einen solchen Verlauf berichteten.

${ }^{13}$ In diesem von G. Rosenthal geleiteten DFG-Forschungsprojekt (RO 827/19-1; https://www.unigoettingen.de/en/477891.html) forschten von März 2014 - Feb. 2019 neben den Autor*innen auch E. Bahl und A. Worm zur Grenze zwischen Marokko und Spanien sowie unsere israelischen Kolleg*innen E. Ben-Ze' ev und N. Gazit zur Grenze zwischen Israel und Ägypten. Siehe u.a. Rosenthal/Bahl/Worm 2016; Ben-Ze'ev/Gazit 2017.

${ }^{14}$ Den Geflüchteten wird entsprechend des so genannten ,deposit law' 20\% des Lohns konfisziert (vgl. https://hotline.org.il/en/information-about-the-new-deposit-law/ <18.07.2019>).

15 Vgl. https://www.haaretz.com/israel-news/israeli-court-suspends-expulsion-of-some-400-congolese-1.6802168<05.03.2019>.
} 
generell gegenüber Vertreter*innen von NGOs oder den Massenmedien. Durchgängig wurde uns erklärt, dass man sich sowohl von Journalist*innen, die Eritreer und Sudanesen in Israel und Uganda ständig zu ihrer Misere befragen wollen würden, als auch von Vertreter*innen von NGOs ausgenutzt fühlen würde. Alle würden einen nur für die eigenen Zwecke benutzen, ohne zu helfen. Wir mussten eingestehen, dass auch wir ihnen - einmal abgesehen von einer Aufwandsentschädigung für das Interview und immer auch verbunden mit einem gemeinsamen Essen in dem Restaurant, das die Befragten jeweils selbst für das Interview vorgeschlagen hatten nicht viel helfen konnten. Neben etlichen ethnographischen Interviews und drei Gruppendiskussionen sowie teilnehmenden Beobachtungen in Cafés und Bars, die von Eritreern besucht werden, konnten wir während unseres dreiwöchigen Aufenthalts in Kampala vier biographische Interviews mit aus Israel ausgewiesenen Eritreern führen und auf Tonband aufzeichnen. Unsere Gesprächsanalysen und Reflexionen über unsere erlebten Interaktionen mit den Eritreer*innen in Kampala lassen uns annehmen, dass gerade unser Deutschsein, unsere nicht beobachtbare Nähe zu Eritrea und unsere Anbindung an eine deutsche Universität zur Öffnung des Dialoges mit uns beitrugen.

Die Sorge vor einem Gespräch über die Fluchtmigration bzw. vor einer Tonaufnahme der Gespräche hängt ganz konkret mit der Asylpraxis und dem Geheimdienst in Uganda zusammen. Die offizielle Leugnung der ugandischen Regierung, mit Israels Regierung ein Abkommen über die Aufnahme von afrikanischen Geflüchteten geschlossen zu haben, erlegt den Abgeschobenen auf, ihren Aufenthalt in Israel und die Abschiebung zu verschweigen. Zudem berichteten unsere Gesprächspartner, dass den eritreischen Flugpassagieren aus Israel alle Papiere, die einen Aufenthalt in Israel belegen könnten, sofort nach Ankunft abgenommen werden (vgl. Birger/ Shoham/Bolzman 2018). ${ }^{16}$ Wenn man einen Antrag auf Asyl stellen möchte, wird man von Angehörigen der Behörden aufgefordert, eine Fluchtroute über den Sudan nach Uganda anzugeben. Dies bedeutet, die „Deportierten“ werden aufgefordert, eine neue Lebensgeschichte zu erfinden und damit das meist über mehrere Jahre andauernde Leben in Israel, die teilweise mit Folter verbundenen extremtraumatisierenden Erfahrungen auf dem Sinai, ${ }^{17}$ den Erwerb von guten hebräischen Sprachkenntnissen, die Erfahrungen mit unterschiedlichen Erwerbstätigkeiten, die in Israel lebenden Freunde, manche auch ihre dort verbliebenen Ehefrauen und die mittlerweile in Israel geborenen Kinder zu verleugnen.

Um die Situation dieser Gruppierung und deren extrem prekäre Situation in Uganda besser zu verstehen, führten wir auch biographische Interviews mit über den Sudan und Äthiopien geflohenen Eritreer*innen (im Folgenden Hadinet und

16 Siehe auch den ausführlichen Bericht von Amnesty International „Forced and Unlawful“ (2018) (https://www.amnesty.org/download/Documents/MDE1584792018ENGLISH.PDF, S. $32<02.03$. 2020>).

17 Vgl. Nakash et al. (2014) sowie detailliert: Human Rights Watch (2014): Human Trafficking in the Sinai: Refugees between Life and Death, https://www.hrw.org/report/2014/02/11/i-wanted-liedown-and-die/trafficking-and-torture-eritreans-sudan-and-egypt $<03.03 .2020>$. 
Simon), die im Unterschied zu den von Israel zur Ausreise genötigten Eritreern einen leichteren Zugang zu Dokumenten haben, die sie sich auf der eritreischen Botschaft ausstellen lassen und somit in einer vergleichsweise etwas etablierteren Situation in Uganda leben können.

\section{Angst vor den Geheimdiensten}

Alle von uns geführten Gespräche verdeutlichten, wie stark die Angst vor den diversen Geheimdiensten ist, wie wenig die aus Eritrea geflohenen Menschen sich wechselseitig trauen und wie deutlich das Misstrauen, die Verschwiegenheit bereits aufgrund der staatlichen Kontrolle in Eritrea internalisiert und Teil des Habitus dieser Menschen ist. Sie gehören zu einer Gruppierung von Menschen, die nie genau wissen können, ob sie überwacht werden und ob sie sich gefährden, wenn sie von sich oder gar ihren in Eritrea zurückgebliebenen Familienangehörigen erzählen (vgl. u.a. Massa 2016). Diese internalisierte Vorsicht wurde deutlich geäußert in einer von uns am Ende unseres Aufenthalts 2018 geführten Gruppendiskussion. Dazu hatten wir unseren Feldassistenten Tom sowie drei zuvor interviewte Eritreer*innen in den Garten unseres Hotels und zu einem anschließenden gemeinsamen Abschiedsessen eingeladen. Neben Tom nahm sein Freund Simon teil. Die beiden Männer kennen sich seit ihrer Kindheit und stammen aus sehr etablierten eritreischen römischkatholischen Familien. Tom ist vom Militärdienst aufgrund einer Erkrankung befreit und lebt legal in Uganda. Simon (Jg. 1988) war aufgrund des Militärdienstes (unter anderem wurde er schwer gefoltert) und den damit verbundenen erschwerten Lebensbedingungen 2011 nach Äthiopien geflohen, konnte dort einen ersten akademischen Abschluss erlangen und reiste 2017 legal nach Kampala, um für ein weiteres Studium von hier aus in ein westliches Land zu gelangen. Die relativ etablierte Situation Simons in Äthiopien hängt neben dem sozialen und ökonomischen Status seiner Herkunftsfamilie vor allem damit zusammen, dass einer seiner Angehörigen zu der „Gruppe der 15“ (G15) gehörte. Diese ehemals ranghohen Offiziere hatten sich 2001 nach dem Krieg mit Äthiopien, als das Regime in Eritrea zunehmend repressiver wurde, für eine demokratische Öffnung des Landes eingesetzt (Hirt/Mohammad 2017). Sie wurden verhaftet; die meisten sind immer noch inhaftiert oder mittlerweile verstorben. ${ }^{18}$

Weiterhin nahm Hadinet (Jg. 1983) an der Diskussion teil. Sie hatte ihr Land aufgrund der politischen Verfolgung ihres Ehemanns verlassen müssen, der nach Deutschland geflohen war. Sie war nach Abschluss ihres Abiturs 2002 mit 19 Jahren zum Militär eingezogen worden und 2011 aus dem Land geflohen. Hadinet gibt im biographischen Interview deutliche Hinweise auf sie traumatisierende Erfahrungen im Militär, erklärt aber explizit, dass sie darüber nicht sprechen kann. Zudem erwähnt sie eine Inhaftierung und Folter während der Militärzeit in der Gruppendis-

18 Die eritreische Regierung gibt bezüglich der G15 nur wenige Details bekannt (vgl. https://www.ecoi.net/de/dokument/1443746.html <13.06.2019>). 
kussion. Hierbei sei nochmals darauf verwiesen, dass Frauen im „Nationaldienst“ häufig von den Kommandeuren sexualisierte Gewalt erleiden müssen und außerdem sehr oft Vergewaltigungen und Prostitution auf der Flucht in Kauf nehmen, um über die jeweiligen regionalen und nationalen Grenzen zu kommen (vgl. Abdulkadir 2018: 54; Leghese 2017). 2011 zwang die Mutter Hadinet zur Heirat mit einem ihr unbekannten Mann. Aufgrund des politischen Engagements ihres Mannes, dessen „Desertion“ 2011 und Flucht über Libyen nach Deutschland war sie selbst wieder von Verhaftung bedroht und floh zur Familie eines Onkels nach Juba im Südsudan. Der Onkel verbot ihr die geplante Fortsetzung der Flucht über das Mittelmeer und gestattete ihr die Migration nach Uganda, wo ebenfalls die Familie eines Onkels lebt. Seit 2011 sitzt sie daher in Uganda fest und wartet darauf, dass es ihrem Mann gelingt, sie nach Deutschland zu holen. Weshalb dies bisher nicht gelungen ist, können wir anhand des Interviews nicht erklären. Deutlich wird nur, dass das Paar - auch mit einem Besuch des Mannes in Kampala - erfolglos versuchte, ein Kind zu zeugen. Deutlich wird auch die enorme Frustration bis hin zu Wut von Hadinet, und es deutet sich in den Gesprächen an, dass sie an der Absicht ihres Mannes zweifelt, sie nachzuholen. Da sie streng katholisch lebt, ist ihr ein Neuanfang mit einem anderen Mann in Uganda nicht möglich. Wie wir kurz vor der Drucklegung dieses Artikels erfuhren, ist ihrem Mann die Ausreise nach Kanada gelungen, er konnte Hadinet nachholen, und sie ist mittlerweile auch schwanger.

Die vierte anwesende Person in der Gruppendiskussion war Robel, der als einziger in dieser Runde zuvor in Israel gelebt hatte. Seine Lebensgeschichte stellen wir im Folgenden noch ausführlicher dar.

Die Gruppendiskussion verhalf uns dazu, das Phänomen besser zu verstehen, dass die Eritreer*innen in Kampala sich einerseits mit uns mehrmals treffen wollten, es auch deutlich wurde, dass sie das Zusammensein mit uns genossen, andererseits jedoch mit einer Zusage für ein biographisches Interview zögerten. Auf der einen Seite wollten sie über ihre gegenwärtige Situation des Gestrandetseins in Kampala sprechen und angenehme Alltagssituationen mit uns „Westlern“ erleben. Auf der anderen Seite fällt es ihnen schwer, ein Gespräch, das auf Tonband aufgenommen wird, mit uns zu führen. Sie müssen achtsam sein, und sie können uns und eigentlich niemandem anderen vertrauen. Und so pendelte jede Begegnung zwischen den Gefühlen von Interesse und Angst sowie zwischen einer durch Vorsicht geprägten Sprechweise und dem Bedarf, über das erlebte Leid zu sprechen. Dies wurde vor allem in der Reaktion auf eine Anmerkung von G. Rosenthal bzgl. der guten Atmosphäre in der Gruppendiskussion deutlich. Alle Teilnehmer*innen äußerten explizit, dass sie sich wechselseitig nicht vertrauen würden. Simon ging so weit und sagte, er traue nicht mal Tom, mit dem er seit seiner Kindheit eng befreundet ist. Robel bekräftigte dies und meinte, mit der deutlichen nonverbalen Zustimmung aller anderen Teilnehmer*innen, dass man nicht mal einem Familienmitglied vertraue. Nicht nur, dass der eritreische Geheimdienst überall auf der Welt ausgesprochen aktiv sei, man nicht wüsste, wer für ihn arbeite, man habe zudem dieses Misstrauen in Eritrea bereits in der Kindheit als Haltung internalisiert, die man auch heute nicht aufgeben 
könne, ganz unabhängig davon, in welchem Land man sich befinde. Denn selbst wenn man außerhalb des Landes etwas Kritisches über Eritrea äußere, könnten Verwandte in Eritrea dafür belangt werden. Dennoch könne man, wie Robel meinte, in Uganda - symbolisch gesprochen - freier atmen, und er wolle keinen Tag mehr in Eritrea verbringen müssen.

Um die Relevanz des eritreischen Geheimdienstes und dessen Wirkmächtigkeit in der Diaspora der Eritreer etwas näher zu beleuchten, werden wir im Folgenden den Fall des ehemaligen Offiziers eines Geheimdienstes vorstellen.

\section{Abraham: Vom Freiheitskämpfer und Geheimdienstoffizier zum in Kampala Gestrandeten}

Abraham (geb. zwischen 1954 und 1956) gehört zu den vier aus Israel ausgewiesenen Eritreern, die G. Rosenthal im Frühjahr 2015 in Kampala interviewte. Er war 2006 über den Sinai nach Israel geflohen, arbeitete in Tel Aviv hauptsächlich als Reinigungskraft bei einem Israeli äthiopischer Herkunft, bis er im August 2014 in Holot interniert wurde. Er beugte sich dem enormen Druck - unter anderem mit der Androhung, ihn nach Eritrea auszuliefern -, der Ausreise schriftlich zuzustimmen, unterschrieb ein Dokument, das er nicht lesen konnte, und wurde mit anderen Eritreern nach Kigali (Ruanda) ausgeflogen. Wie auch die anderen 2015 von der Autorin interviewten Männer wurde er in einem Hotel untergebracht und erhielt beim Aufsuchen des Immigrationsbüros ein Touristenvisum für drei Wochen. Gleich bei Ankunft in Kigali wurde ihm und den anderen der Kontakt zu einem Schlepper vermittelt, der sie ,illegal“ bei Nacht über die Grenze nach Uganda bringen könne. Der Weg bis nach Kampala kostete ihn 3.500 US-Dollar - genau die Summe, die ihm in Israel zur Unterstützung seiner Ausreise gezahlt worden war.

Dieser Verlauf gleichwie seine traumatisierenden Erlebnisse auf der Route durch den Sinai sind sehr ähnlich wie die Geschichten, die wir aus der Literatur kennen (vgl. Fußnote 16). Doch Abrahams Vergangenheit in Eritrea, seine Position in der Community der Eritreer und seine gegenwärtige Situation unterscheiden sich - so vermuten wir - von allen anderen sowohl in Israel als auch in Uganda interviewten Eritreer*innen. Da wir davon erst etwas mehr im dritten und vor allem im vierten Gespräch mit Abraham erfuhren, müssen wir einschränkend vermerken, dass wir nicht wissen können, was sich hinter den Selbstpräsentationen anderer Befragter verbirgt, was uns verschlossen blieb. Abraham berichtete erst im vierten Interview, das von Tom allein geführt wurde, explizit über seine hohe Position in der eritreischen Armee, seine Nähe zum Diktator Isayas Afewerki und seine Geheimdiensttätigkeiten vor allem im Sudan. Auch meinte Abraham zu Tom: „I can share some secrets, but I have to unrevealed some secrets as well ${ }^{\text {“19 }}$ und sprach z.B. darüber,

${ }^{19}$ Alle Gespräche mit Abraham sind in seiner Muttersprache Tigrinya geführt und von Tom ins Englische übersetzt worden. Die Zitate sind nicht die Übersetzungen im Interview, sondern die sehr 
dass er Rebellen aus dem Jemen in Eritrea trainiert habe: ,We were training Yemenis this was the nature of our work. Now since we are kept as secret, we had Somalis, the ones from Sudan, Darfur." Diese Information muss vor dem Hintergrund gesehen werden, dass die Regierung von Eritrea immer wieder - u.a. 2010 - abstritt, Rebellen aus anderen Ländern, wie dem Iran oder dem Jemen, in Eritrea zu trainieren und angab, es handele sich dabei um westliche Gerüchte (vgl. Shaheen 2010). Im fünften Gespräch im Januar 2016 bot Abraham auch G. Rosenthal den Verrat von internen eritreischen Staatsgeheimnissen an, sollte sie ihm die Ausreise nach Deutschland ermöglichen - ein Angebot, das die Forscherin nicht annehmen konnte.

In den ersten zwei Interviews hielt sich Abraham noch sehr bedeckt. Seine Erzählungen konzentrierten sich hier vor allem auf die erlebte Entführung und Folter auf dem Sinai, dem damit zusammenhängenden ökonomischen Ruin für sich und seine Familie, die für die Lösegeldsumme (er spricht zunächst von 25.000, später immer wieder von 40.000 US-Dollar) Grundbesitz verkaufen musste. Er erzählt über die Organentnahme ${ }^{20}$ durch Ärzte, die alle Weiße gewesen seien und Arabisch gesprochen hätten, wie er miterlebte, dass Menschen nach der Entnahme von Organen starben und von den Leichenbergen: ,You see the harvested bodies one top of the other thrown there."

Seine hohe Position im Militär deutete sich zunächst nur etwas zwischen den Zeilen an, und er versuchte seine Migration nach Israel als aus ökonomischen Gründen motiviert darzustellen - ein Motiv, das durch die späteren Gespräche zwar bestätigt wurde, doch mit einer völlig anderen Rahmung. Im ersten biographischen Interview eröffnete er seine biographische Erzählung wie folgt:

"Ok, my name is Abraham B. and I fought for the liberation of Eritrea for twenty-five years. And the reason I went to Israel is due to my economically situation at home. See, I support both my children and the children of my brothers who died during the war for independence. So I left the Eritrean government and went to Israel to solve my economic situation ..."

Diese Darstellung durchzieht das gesamte erste Interview, obgleich bereits in dieser ersten Sequenz ein Hinweis auf seine Position in Eritrea zu finden ist. Er spricht nicht davon, dass er Eritrea verließ, sondern die eritreische Regierung. Diesen Hinweis - vermutlich völlig unbeabsichtigt - gab er auch in einer späteren Sequenz, in der er wiederholt, er sei aus ökonomischen Gründen weggegangen. Er schließt dies mit folgender Evaluation ab: „And of course I'm not pleased with what the government is doing in Eritrea and it pains me inside."

genauen Übersetzungen seiner auf Tonband aufgenommenen Aussagen durch Tom, die wir ohne Korrekturen wiedergeben.

${ }^{20}$ Zum Organhandel auf dem Sinai vgl. den Bericht der Schweizerischen Flüchtlingshilfe von 2012: https://www.refworld.org/pdfid/58514b2e4.pdf <02.09.2020>. Ebenso der Bericht von Pro Asyl von 2013: https://www.proasyl.de/news/fluechtlinge-im-sinai-opfer-von-sklaverei-loesegelderpressung-und-organentnahme/ $<02.03 .2020>$. 
Das zweite Gespräch im Rahmen eines Besuchs bei einem Notar in Kampala diente dazu, ein Affidavit zu erstellen, in dem Abraham über die Nötigung zur Ausreise aus Israel und deren Verlauf, einschließlich der Ausweisung aus Ruanda, Zeugnis ablegte. G. Rosenthal hatte ihn um diese notariell bestätigte Aussage gebeten, da unsere israelischen Kolleg*innen dies einer zivilgesellschaftlich aktiven Anwältin für ein Gerichtsverfahren gegen die Ausweisepolitik der israelischen Regierung zur Verfügung stellen wollten. In diesem Affidavit gibt Abraham an, dass er in Eritrea gegen einen Vorgesetzten im Militär opponiert habe, deshalb in das Gefängnis kam und von dort aus floh. Der Notar bat ihn um den Namen des Gefängnisses, und mit der sehr zögerlichen Nennung des Namens, auf mehrmaliges Insistieren hin, wurde deutlich, dass es sich um ein Gefängnis für hohe Offiziere handelt, die dort in der Regel ohne Gerichtsverfahren inhaftiert werden. Die Anwaltskanzlei in Israel war mit den wenigen Details in diesem Affidavit nicht zufrieden und bat um ein detaillierteres, das von Anwälten einer NGO in Kampala erstellt werden sollte. Abraham willigte ein, und nur wenige Monate später gab er in diesem Kontext die Information, dass er aufgrund seines Protests gegen die Lebensbedingungen und das minimale Gehalt beim Militär gegenüber seinem „Vorgesetzten“ („Commander") zunächst inhaftiert und nach zwei Monaten zum Tode verurteilt worden war. Aufgrund dieser Information bat G. Rosenthal Tom, mit Abraham ein weiteres biographisches Interview zu führen. Erst nachdem Abraham den Familienhintergrund von Tom überprüft hatte, auch erfahren hatte, wer genau Toms Vater ist, ihn im wahrsten Sinne des Wortes verhört hatte, war eine Basis des Vertrauens geschaffen, und er stimmte einem weiteren Interview und auch der Aufnahme des Gesprächs auf Band zu. Er sprach in diesem Interview von seinem hohen Offiziersrang, einem Treffen im Frühjahr 2006, das er gemeinsam mit anderen ranghohen Offizieren mit dem Präsidenten Afewerki persönlich hatte, ihrem Beklagen des geringen Gehalts, ihrer Inhaftierung (er gibt dabei alle Namen seiner Mitgefangenen an), seiner Flucht aus dem Gefängnis und der Hinrichtung aller mit ihm inhaftierten Offiziere.

Im Verlauf der zunehmenden Informationspreisgabe von Gespräch zu Gespräch wird deutlich, dass Abraham immer bei der Wahrheit blieb und nur durch Weglassen von Informationen ein etwas anderes Bild über seine Flucht aus Eritrea vermittelte. Er wurde aus seiner Perspektive aus ökonomischen Gründen zum Tode verurteilt und musste deshalb aus Eritrea flüchten. In der Antwort auf die Frage von Tom nach den Motiven für die Fluchtroute nach Israel und nicht nach Europa erläuterte er ganz offen, dass der Weg nach Libyen für ihn viel zu gefährlich war und erklärte nochmals sehr offen seine Rolle im Militär:

"So I couldn't go to Khartoum; because since I am now one of the wanted officials given that from those we were secretly training and our unit had direct communications I couldn't go to Khartoum. I could not go to Ethiopia; because we were training the opposition and I was in the borders monitoring unit, spying division, they know me by name, they know what my job is." 
Abraham führt weiter aus, dass er bereits während des Unabhängigkeitskrieges Mitglied einer Einheit des Geheimdienstes war, dies auch nach 1991 blieb, in diesem Zusammenhang im Südsudan die Befreiungsarmee „Sudan People's Liberation Army“" (SPLA) trainierte und nach dem Friedensabkommen der SPLA mit der sudanesischen Regierung von 2005, mit dem der Südsudan (zunächst) zur autonomen Region wurde, zuletzt in Darfur bis zum Friedensabkommen für Darfur im Mai 2006 eingesetzt war. Zu dieser Zeit fand das Treffen mit Afewerki statt.

In welcher Situation befindet sich heute dieser ehemals ranghohe Offizier des eritreischen Militärs im Alter von über 60 Jahren, der zudem erhebliche Verletzungen aus dem Unabhängigkeitskrieg davontrug, Schwierigkeiten beim Gehen hat und dessen Frau und Kinder in einer ökonomisch prekären Situation in Eritrea leben? Wir können davon ausgehen, dass er, abgesehen von Gelegenheitsjobs in Israel, nie außerhalb des Militärs gearbeitet hat. Mit der Angabe, dass er 25 Jahre für die Unabhängigkeit Eritreas gekämpft habe, verweist er auf den mit Unterbrechungen stattgefundenen Unabhängigkeitskrieg, der 1961 begann und erst 1991 mit der Unabhängigkeit des Landes endete. Damit war er zu Beginn dieser Zeit noch ein Kind. Je nachdem, wann er geboren ist (hier macht er widersprüchliche Angaben), war er mit Eintritt in die damalige Rebellenarmee zwischen 10 und 12 Jahre alt; auch wenn dies nicht bedeutet, dass er damit bereits in den ersten Jahren in einer kämpfenden Einheit der Eritrean Liberation Front (ELF) oder ab 1970 der sich von der ELF abgespaltenenen Eritrean People's Liberation Front (EPLF 1970) eingesetzt wurde. ${ }^{21}$ Für dieses junge Alter spricht auch, dass er im Unterschied zu seinen vier älteren Brüdern, die alle in diesem Krieg getötet wurden, über keine Schulbildung verfügt.

Aufgrund seiner Geheimdiensttätigkeiten im Sudan ist es für ihn viel zu gefährlich - wie er selbst ausführt -, Uganda zu verlassen. Damit ist er im wahrsten Sinne des Wortes in Kampala gestrandet. Er erhielt zwar bereits im Februar 2015 ein temporäres Aufenthaltsrecht als Asylsuchender, doch dieses muss immer wieder erneuert werden. Dennoch ist seine Situation im Vergleich zu den anderen Gestrandeten etwas besser. Zum einen kennt er sehr genau die Methoden des eritreischen Geheimdienstes, wusste auch in Israel, dass er überwacht wurde. Er erläutert, dass er wisse, in welchen Ländern man sicher ist und in welchen nicht, und er fühle sich in Uganda einigermaßen sicher. Solange er nicht öffentlich Kritik gegen das Regime in Eritrea äußert, würden sie ihn nicht umbringen, erklärt er der Autorin. Würde er sich dagegen im Sudan aufhalten, dann würde er ermordet werden. Er kenne das eritreische System von innen und von außen, bekräftigt er im letzten Gespräch. Er habe auch genügend Kontakte in Eritrea, um zum Beispiel regelmäßig mit seiner Frau zu telefonieren, ohne dass die eritreischen Behörden dies mitbekommen.

Insgesamt verdeutlicht diese Fallstudie, wie sehr Abrahams in Eritrea vorhandenes Prestige auch noch heute handlungswirksam für andere Eritreer ist. Abraham

\footnotetext{
${ }^{21}$ Selbst wenn er zu den 25 Jahren auch die zwei Jahre Krieg (1998-2000) mit Äthiopien dazurechnet, wäre er beim Einzug zur Armee erst 14 Jahre alt gewesen. Zum Einsatz von Kindersoldaten im Unabhängigkeitskrieg vgl. den UNHCR Bericht von 2001: https://www.refworld.org/docid/498805fd5.html <07.03.2019>.
} 
kommt aus einer etablierten gesellschaftlichen bzw. politischen Position und kann sich - wie sich im Zusammensein mit ihm und anderen Eritreern in Uganda deutlich zeigte - auf weiteren Respekt in der Gruppierung der eritreischen Geflüchteten und auf deren Hilfeleistung auch in Uganda (z.B. der kostenlosen Überlassung eines Hotelzimmers) verlassen. ${ }^{22}$ Er kann sich darauf verlassen, obwohl die anderen Geflüchteten aus der von ihm sehr geschätzten eritreischen Armee desertiert sind und er vermutlich früher die Inhaftierung von Deserteuren in Eritrea für richtig hielt, wenn nicht sogar selbst anordnete. Abraham verfügt weiterhin über etliche Verbindungen zu Angehörigen der politischen Eliten in Eritrea und über die Kompetenz geheimdienstlicher Ermittlungen bzw. Verhörmethoden. Seine Hoffnung, aus der gegenwärtigen Situation herauszukommen, gründet sich auf Optimismus über einen erhofften Sturz des eritreischen Präsidenten Afewerki. Er verortet sich in diesem Zusammenhang in dem „Wir“ der langjährigen Freiheitskämpfer: „We are the ones who fought for and brought liberty there. We could not just stay by and watch these idiots manipulate it ... result is the knife". Diese Ankündigung von Widerstand, der seiner Ansicht nach nur von innen kommen kann, wird noch dadurch bekräftigt, dass er zu Tom meint: „Tell her (G. Rosenthal) that I would one day live in my country, I will work in a civilian job and I believe I will be once again in Eritrea."

\section{Robel: Auf der ständigen Suche nach einem Land mit Bleiberecht}

„If you are staying there [in Israel] you are immigrant it means you are beggar, a beggar could not choose nothing, but I hate my choice“, äußert Robel (Jg. 1977) in einem auf Englisch geführten Interview mit L. Hofmann. M.a.W., Robel möchte kein Bettler sein, er möchte in einem Land leben können, in dem er das Recht auf Bleiben und damit auch auf Zugehörigkeit hat. Die Lebenssituation eines Immigranten bestimmte bereits seine Jugend, da er und seine Familie zu jenen Eritreern gehören, die $1998 \mathrm{im}$ heutigen Staatsgebiet von Äthiopien lebten, enteignet und nach Eritrea ausgewiesen wurden. Die Familie war in Äthiopien gut situiert, der Vater arbeitete zunächst als Mechaniker und dann als Fahrer für eine UN-Organisation. Die sechs Kinder der Familie hatten bis zur Ausweisung alle eine gute Schul- und Ausbildungslaufbahn eingeschlagen. Doch seit 1998 lebt die Familie in einer prekären Situation. Alle Kinder wurden zum Militär zwangsverpflichtet, der Vater wurde mehrmals inhaftiert, und ein Bruder Robels ist im eritreischen Militär gestorben. ${ }^{23}$

\footnotetext{
22 Da G. Rosenthal Abraham in verschiedenen Situationen zusammen mit anderen Eritreern traf, in denen sie zum eritreischen Kaffeeritual eingeladen war, deckt sich diese aus den Interviews gewonnene Annahme auch mit den Befunden der teilnehmenden Beobachtungen.

${ }^{23}$ Dieser lebens- und familiengeschichtliche Verlauf: 1998 Zwangsverpflichtung zum eritreischen Militär, Abbruch der in Äthiopien begonnenen Bildungs- und Berufswege und die ökonomische Prekarität der Familien ist ein sehr typischer für die Generation von Robel und für seine Familie insgesamt (vgl. Kibreab 2017a: 128 ff.).
} 
Der jüngste Bruder floh wie Robel nach Israel und lebt noch heute dort, seine beiden Schwestern leben weiterhin in Eritrea.

Wir trafen Robel im Februar 2018 in Kampala, wo er seit fast drei Jahren lebte. Er hatte sich 2015 in Israel dem Druck der Behörden zur Ausreise gebeugt, obwohl er seine Frau, seine beiden Kinder und seinen Bruder zurücklassen musste. ${ }^{24}$ Vor diesem Hintergrund ist es nicht erstaunlich, dass er im ersten Interview fast ausschließlich über seinen unermüdlichen Kampf für eine Anhörung vor Gericht in Uganda berichtete, in der er seine Deportation aus Israel anerkannt haben möchte, um damit auch einen Asylstatus beantragen zu können. Dafür sucht er unbeirrt den Kontakt zu NGO-Vertreter*innen und versucht - auch mit Bestechungen -, die benötigten Nachweise über seine Ausreise aus Israel von ugandischen Beamten ausgestellt zu bekommen. Er ist keineswegs bereit, sich der Auflage zu beugen, seine Vergangenheit in Israel zu verschweigen, weil damit der Nachzug seiner Gründungsfamilie gefährdet sein könnte.

Es bedurfte mehrerer Treffen, bevor sich Robel für ein biographisches Interview bereitfand, das von L. Hofmann an zwei weiteren Terminen durchgeführt wurde. Trotz der Bitte, seine Familien- und Lebensgeschichte zu erzählen, spricht er in beiden Gesprächen vor allem über seine aktuelle Situation und erzählt nochmals im Detail über seinen Kampf um eine Anhörung vor Gericht in Uganda. Die Analyse dieser Gespräche verdeutlicht, dass er erheblich mit Schuldgefühlen gegenüber seiner Gründungs- und Herkunftsfamilie zu kämpfen hat. Er fühlt sich verpflichtet, alles dafür zu tun, um deren Situation zu verbessern. Damit unterscheidet er sich von jenen von uns Interviewten, die eher wie gelähmt in der Aussichtslosigkeit verharren. Allerdings müssen wir uns auch fragen, wie lange Robel die Suche nach Auswegen aus der gegenwärtigen Lebenssituation - die sich durch die Trennung von seiner Gründungsfamilie auszeichnet - durchhält.

Der familien- und lebensgeschichtliche Verlauf dieses Geflüchteten verdeutlicht, wie er von Fluchtmigration geprägt ist. Der Fall repräsentiert den leidvollen Verlauf einer Familie, die zur ethnischen Gruppierung der Tigray ${ }^{25}$ gehört und aus dem Gebiet der bis 1941 bestehenden italienischen Kolonie Eritrea stammt. Die Eltern von Robel sind Anfang der 1960er Jahre, also in der Zeit, in der die autonome Region Eritrea von Äthiopien annektiert wurde, in die Hauptstadt Äthiopiens zum Arbeiten gezogen, wo auch ihre sechs Kinder geboren wurden. Die Familie erlebte damit eine politisch sehr unruhige Zeit, geprägt durch den seit 1961 andauernden Kampf gegen die Annexion, Dürren in den 1970er Jahren und einen Militärputsch 1974 in Äthiopien. Es folgten weitere Destabilisierungen in der Region durch den

242015 wurden ca. 485 Eritreer und Sudanesen nach Uganda ausgeflogen (vgl. https://observer.ug/news/headlines/57721-uganda-now-opts-out-of-israel-migrants-relocation-deal.html $<24.11 .2018>$ ).

${ }_{25}$ Diese Gruppierung lebt im Norden Äthiopiens, in der Provinz Tigray, und im angrenzenden Eritrea. In Eritrea bilden sie die größte Bevölkerungsgruppe mit ca. 50\%, in Âthiopien ca. 5\%. Die Bezeichnung dieser Ethnie variiert je nach Land, in Eritrea werden sie Tigrinya und in Äthiopien Tigray genannt (vgl. Appleyard 2017: 46 ff.). 
Krieg mit Somalia 1977/1978, die Dürre 1984/1985, in der über eine Million Menschen starben, sowie Repressionen und Tötungen von Oppositionellen. Die Entmachtung des äthiopischen Diktators Mengistu Haile Mariam 1991 führte zu einem kollektivgeschichtlich entscheidenden Wendepunkt, der sieben Jahre später zur Enteignung und Deportation von Robels Familie führte. Der dreißigjährige - mit Unterbrechungen geführte - Unabhängigkeitskampf der Eritreischen Befreiungsfront, später der von dieser abgespalteten Eritreischen Volksbefreiungsfront, endete mit der Bildung einer neuen äthiopischen Regierung und ermöglichte die Unabhängigkeit Eritreas im Mai 1993. Doch bereits im Mai 1998 begann ein zweijähriger Krieg zwischen Äthiopien und Eritrea, worauf die äthiopische Regierung die Enteignung und Deportation von 200.000 in Äthiopien lebenden Eritreer*innen nach Eritrea veranlasste (vgl. Hunziker 2000: 8). Robel war damals 21 Jahre alt, hatte einen Abschluss der High School, ein Jahr eine Commercial School in Addis Abeba besucht und strebte ein Universitätsstudium an. Doch in Eritrea wurde er, wie seine Schwestern und seine Brüder, sofort ins eritreische Militär eingezogen. Wie sehr dieser biographische Wendepunkt seine Ambivalenz gegenüber Eritrea bestimmt, zeigt sich in seiner Selbstpositionierung, die Robel im ersten Interview wie folgt auf den Punkt bringt:

"I am born in Ethiopia I grow there I don't I have anything to study in Eritrea but I grow up in Ethiopia and I served in Eritrea."

Robel gehört zu jenen Eritreern, die durch ,ihre Geburt und ihr Aufwachsen“ in Äthiopien, wie es Robel selbst formuliert, mit erheblichen Ambivalenzen hinsichtlich der nationalen Zugehörigkeit zu kämpfen haben, insbesondere dann, wenn sie als Soldaten des eritreischen Militärs an kriegerischen Auseinandersetzungen teilnehmen mussten. Zunächst identifiziert mit den Zielen des Kampfes für die Unabhängigkeit Eritreas - wie es auch Robel deutlich zum Ausdruck bringt -, waren sie im Krieg mit Äthiopien jedoch in der Situation, Menschen zu töten, die teilweise früher zu ihren Bekannten, Freunden oder auch Verwandten gehörten.

Nach zwei Jahren des Militärdienstes in Eritrea, also im Jahr 2000, endete zwar der Krieg mit Äthiopien, doch Robel wurde nicht entlassen. 2003 entschied sich Robel zur Desertion und fand eine Anstellung bei einem international tätigen Unternehmen. Dies ermöglichte ihm, seine Familie finanziell zu unterstützen, doch als Deserteur musste er ständig mit einer Verhaftung rechnen. Dies geschah auch nach eineinhalb Jahren, und Robel wurde zu einer zwölfjährigen Haftstrafe verurteilt. Nach einem Monat gelang ihm die Flucht mit seinem jüngeren Bruder in den Sudan. In Khartum lebte Robel drei Jahre, eröffnete ohne rechtliche Genehmigung ein Hostel und lernte seine spätere Frau Sarah kennen, die ebenfalls zu den aus Äthiopien nach Eritrea Deportierten gehört sowie aus dem „Nationaldienst“ geflohen war. Sarah wurde 2007 schwanger und entschied sich zur Flucht nach Israel, wohin bereits Robels Bruder migriert war. Robel folgte ihr zwei Monate später, als ihr erstes Kind bereits geboren war. 
Fünf Jahre arbeitete Robel als Reinigungskraft in wechselnden Gaststätten. Zu der Zeit wurden in Israel Arbeitskräfte gesucht und auch ohne eine offizielle Arbeitserlaubnis immer wieder für kurze Zeit eingestellt. Das Leben für Robel und Sarah stabilisierte sich ein wenig, und sie bekamen ihr zweites Kind. Durch die Notwendigkeit, jeden oder jeden zweiten Monat auf der Behörde das Visum verlängern lassen zu müssen, ${ }^{26}$ stand aber die Gefahr einer Ausweisung ständig im Raum. Der damit einhergehende unsichere Zukunftshorizont verstärkte sich für Robel, wie auch für viele andere geflüchtete Afrikaner, durch das 2013 eröffnete Internierungslager Holot, den beginnenden Druck der Behörden zur Zustimmung zu einer Abschiebung und die fast vollständige Aussichtslosigkeit einer Bewilligung eines ab 2013 möglichen Antrags auf Asyl. ${ }^{27}$ Eine aussichtsreich erscheinende Migration nach Italien scheiterte für ihn und seine Familie. Die von Bekannten in Italien besorgten Visa erwiesen sich bei der Ausreise als Fälschungen. Vor diesem Hintergrund entschied sich Robel Ende 2015, am israelischen Ausweisungsprogramm teilzunehmen und sich in einen, sicheren' afrikanischen Staat deportieren zu lassen - mit der Hoffnung, seine Familie nachholen zu können. Im Flugzeug erfuhr er, dass er und die sechs anderen „Deportierten“ in Entebbe, Uganda, landen werden. Dort drohte ihnen, wie Robel bereits aus mehreren Berichten und Veröffentlichungen wusste, die Abnahme ihrer Ausweispapiere. Es gelang ihm, seine Papiere aus dem Flughafen „zu schmuggeln“. Er berichtet darüber, dass er und die anderen Deportierten durch einen separaten Ausgang aus dem Flughafen geschleust und für eine Nacht in ein Hotel einquartiert worden seien. Am nächsten Tag sei ihnen von ugandischen Beamten erklärt worden, dass sie Uganda zu verlassen haben und in „ihre Länder“ zurückgehen sollen. Sollten sie dennoch zu bleiben beabsichtigen, so dürften sie in Uganda nichts über ihre Abschiebung aus Israel erzählen. Damit wurden Robels Hoffnungen auf ein Bleiberecht in einem afrikanischen Land und auf die Möglichkeit, seine Familie nachholen zu können, zerstört. Er bringt es im Interview auf den Punkt: „In Uganda things became broken.“ Er begann über Möglichkeiten nachzudenken, dieser aussichtslosen Situation zu entkommen. Viele, wie auch die von G. Rosenthal in 2015/2016 in Kampala interviewten Eritreer, schlagen den sehr gefährlichen Weg nach Libyen und über das Mittelmeer nach Europa ein, obwohl ihnen die Gefahren, vor allem auch durch die jihadistische Miliz IS und andere Rebellengruppierungen, durchaus bekannt sind. Doch für Robel war diese gefährliche Route als Familienvater keine Option mehr: „I don't won't to get in this kind of risk, because I know the risk ... I have kids now". Stattdessen versuchte er, ein Jahr nach seiner Ankunft in Uganda, mit einem gefälschten Pass nach Kanada zu migrieren. Er reiste mit dem Flugzeug über Khartum nach Doha und wurde dort jedoch aufgrund des gefälschten Passes verhaftet. Da die Beamten ein eritreisches Ausweisdokument bei ihm fanden, wurde ihm mit der Abschiebung nach Eritrea gedroht.

\footnotetext{
${ }^{26}$ Seit Feb. 2008 wurden noch ein bis zwei Monate gültige „Conditional Release Visas“ ausgestellt. Zuvor bestand Anspruch auf für ein Jahr gültige Papiere (vgl. Birger/Shoham/Bolzman 2018: 16).

27 Die Anerkennungsquote liegt bei $0,48 \%$ (vgl. https://hotline.org.il/wp-content/uploads/2018/10/Eng-Web-RSD-Report-HRM-17Oct2018.pdf, 9 f. <29.05.2019>).
} 
Robel erzählt, er habe erreicht - u.a. mit Suizidandrohungen und aufgrund seiner Arabischkenntnisse -, dass er nach Khartum zurückfliegen durfte. Da der Sudan keineswegs eine bessere Option ist, kehrte er nach Kampala zurück.

Seine Versuche, in ein Land zu migrieren, das ihm und später seiner Frau und seinen Kindern ein Bleiben ermöglicht, konfrontieren ihn immer wieder mit der Gefahr, nach Eritrea abgeschoben und dort inhaftiert zu werden. Diese Gefahr ist ein zentraler Bestandteil seines Lebensgefühls, und so sieht er in der Gegenwart in Uganda für sich nur eine Handlungsoption: Er muss vor Gericht gehen und sein Recht einklagen, dass er als aus Israel ausgewiesener Eritreer Asyl beantragen und seine Gründungsfamilie nachholen kann. Der damit verbundene Aktivismus gibt ihm das Gefühl, handlungsmächtig zu bleiben.

\section{$6 \quad$ Fazit}

Vergleichen wir die Situation dieser beiden Männer, die eine sehr unterschiedliche gesellschaftliche Stellung in Eritrea besetzten und auch gegenwärtig in Kampala einnehmen, eine sehr divergente Haltung zum politischen System in Eritrea hatten sowie aus verschiedenen Gründen ihr Land verließen, so zeigen sich in der Gegenwart doch deutliche Ähnlichkeiten. Sie repräsentieren einen Typus von Geflüchteten, den man als „Illusionen über und Aktivitäten für eine bessere Zukunft“ bezeichnen kann. Im Unterschied zu Repräsentanten des Typus „Verloren in Passivität und Aussichtslosigkeit" erhalten Abraham und Robel mit viel Energie ihre Handlungsmacht und ihr Selbstwertgefühl in einer von außen betrachtet nahezu aussichtslosen Lebenssituation aufrecht. Beide hoffen auf eine deutlich veränderte Zukunft und verharren nicht in Passivität, wie die meisten anderen von uns interviewten Eritreer*innen, sondern versuchen, sich für die erhofften Veränderungen aktiv einzusetzen. Während Abraham zurück nach Eritrea und damit auch zu seiner Frau, seinen Kindern und deren Familien möchte, sucht Robel nach einem Land, in dem er und seine Familie Bleiberecht bekommen können. Abraham verweist auch mit der Aussage „we could not just stay by and watch this idiots manipulate it" darauf, dass er selbst aktiv zum Ende der Herrschaft der „Idioten“ beizutragen versucht. Und Robel gibt den Versuch nicht auf, sich vor Gericht, also auf legalem Weg, in Uganda Recht zu verschaffen und seine Familie nachholen zu können. M.a.W., der seit langem etablierte Plan für die Zukunft, zu dem zentral auch die Wiedervereinigung mit der Kernfamilie gehört, ist ein konstitutiver Bestandteil ihrer Handlungsplanung in der Gegenwart.

Eine weitere Ähnlichkeit der biographischen Verläufe und Fluchtmigrationen dieser beiden Männer ist, wie für viele Geflüchtete, das hilflose Ausgeliefertsein an die Folgen von zwischenstaatlichen Abkommen, unter anderem durch die Europäische Union initiiert, und an die gesellschaftlichen Entwicklungen in mehreren afrikanischen Ländern. Die Bestimmungen dieser Abkommen sind oft unberechenbar, oft willkürlich und rechtlich sowie moralisch extrem problematisch. Im behandelten 
Fall ist dies besonders deutlich, u.a. was die Abkommen zwischen Israels und Ugandas Regierung, die damit verbundenen Schwierigkeiten, in Uganda als Asylsuchender anerkannt zu werden, die Aufforderung, die Lebenszeit in Israel zu verleugnen, sowie besonders, was die Behandlung bzw. Diskriminierung von Asylsuchenden aus Eritrea betrifft - einem Land, das in den Medien immer wieder mit dem heutigen Nordkorea verglichen wird, also einem extrem undemokratisch regierten und hinsichtlich der Menschenrechtslage höchst kritikwürdigen Staat.

So stellen sich für die von uns vorgestellten beiden Männer wie auch für andere aus Eritrea geflüchtete Menschen Fragen an eine von ihnen kaum beeinflussbare Zukunft: Bleibt Eritrea ein Land, in dem sie sich nie wieder ohne Lebensgefahr aufhalten können? Bleibt Uganda ein Land, aus dem man nicht ausgewiesen wird? Eröffnen sich Lebenschancen in den umliegenden Ländern - wäre z.B. Äthiopien eine Option für Robel oder der Sudan für Abraham oder könnten sie sich (auch mit ihren Kernfamilien) in Uganda etablieren?

\section{Literatur}

Abdulkadir, A. (2018): Situation von Frauen und Mädchen in Eritrea. In: Pro Asyl: Eritrea. Frankfurt a. M.: Förderverein Pro Asyl e.V., 53-55.

Appleyard, D. (2017): Tegray. In: Uhlig, S./Appleyard, D./Bausi, A./Hahn, W./ Kaplan, S. (Hrsg.): Ethiopia. Münster: Lit-Verlag, 46-49.

Bartolucci, B. (2017): Wehrdienst ist nicht gleich Wehrdienst. Wie Flüchtlingen aus Eritrea der Schutz verwehrt wird. In: Pro Asyl: Tag des Flüchtlings. Frankfurt a. M.: Förderverein Pro Aysl, 20-22.

Becker, D. (2007): Die Erfindung des Traumas. Verflochtene Geschichten. Berlin: Ed. Freitag.

Ben-Ze'ev, E./Gazit, N. (2017): Situational Humanitarianism. Israeli Soldiers and Asylum Seekers on the Egyptian-Israeli Border (Article in Hebrew). In: Hagira - Israel Journal of Migration, 7, 84-101.

Birger, L./Shoham, S./Bolzman, L. (2018): "Better a Prison in Israel than Dying on the Way”. Online: http://assaf.org.il/en/content/better-prison-israeldying-way-testimonies-refugees-who-voluntarily-departed-israel-rwanda $<02.09 .2020>$.

Bogner, A./Rosenthal, G. (2018): KindersoldatInnen im Kontext. Biographien, familien- und kollektivgeschichtliche Verläufe in Norduganda. Göttingen: Universitätsverlag Göttingen. Online: https://doi.org/10.17875/gup2018$1122<09.04 .2020>$.

Hirt, N. (2016): Flüchtlinge aus Eritrea. Spielball europäischer Interessen. Hamburg: GIGA. 
Hirt, N./Mohammad, A. S. (2017): By Way of Patriotism, Coercion, or Instrumentalization. How the Eritrean Regime Makes Use of the Diaspora to Stabilize Its Rule. In: Globalizations, 15(2), 232-247.

Hirt, N./Mohammad, A. S. (2018): The Lack of Political Space of the Eritrean Diaspora in the Arab Gulf and Sudan. Torn Between an Autocratic Home and Authoritarian Hosts. In: Journal of Middle East Migration Studies, 5(1), 101126.

Hunziker, P. (2000): Deportation ethnischer Minderheiten aus Äthiopien und Eritrea. Bern: Schweizer Flüchtlingshilfe. Online: https://www.ecoi.net/en/file/local/1002680/470_tmpphpCWyM33.pdf $<20.11 .2018>$.

Keilson, H. (1979): Sequentielle Traumatisierung bei Kindern. Stuttgart: Enke.

Kibreab, G. (2017a): The Eritrean National Service. Servitude for "the Common Good" and the Youth Exodus. Woodbridge: James Currey.

Kibreab, G. (2017b): Sexual Violence in the Eritrean National Service. In: African Studies Review, 60(1), 123-143.

Leghese, M. (2017): A Study of Impacts of Diversity among Female Youth Eritrean Refugees. Unv. BA-Thesis. Addis Abeba: Ethiopian Catholic University of Saint Thomas Aquinas.

Massa, A. (2016): Learning Not to Ask. Some Methodological Implications of Studying Eritrean Refugees in Ethiopia. In: Zeitschrift für Ethnologie, 141, 257-280.

Mekonnen, D. R./Estefanos, M. (2012): From Sawa to the Sinai Desert. The Eritrean Tragedy of Human Trafficking. Online: https: / ssrn.com/abstract $=2055303<06.03 .2019>$.

Nakash, O./Langer, B./Nagar, M./Shoham, S./Lurie, I./Davidovitch, N. (2014): Exposure to Traumatic Experiences Among Asylum Seekers from Eritrea and Sudan During Migration to Israel. In: Journal of Immigrant and Minority Health. Online: https://doi.org/10.1007/s10903-014-0022-y <09.04.2020>.

Rosenthal, G. (2015): Interpretative Sozialforschung. Eine Einführung. 5. Auflage. Weinheim: Beltz Juventa.

Rosenthal, G./Bahl, E./Worm, A. (2016): Illegalisierte Migrationsverläufe aus biografietheoretischer und figurationssoziologischer Perspektive. Die Landgrenze zwischen Spanien und Marokko. In: Forum Qualitative Sozialforschung, 17(3), Art. 10. Online: www.qualitativeresearch.net/index.php/fqs/article/view/2686/4012<05.05.2020>. 
Shaheen, A. N. (2010): Eritrea Denies Training Rebels for Iran and Yemen. Online: https://gulfnews.com/world/mena/eritrea-denies-training-rebels-foriran-and-yemen-1.615399<07.03.2019>.

Tesfagiorgis, M. (2013): From Troubled Homes to Human Organ Harvesters. In: Mekonnen, D. R./Tesfagiorgis, M. (Hrsg.): The Horn of Africa at the Brink of the 21st Century. Felsberg: Edition Eins, 63-100.

Treiber, M. (2013): Grasping Kiflu's Fear. Informality and Existentialism in Migration from North-East Africa. In: Modern Africa. Politics, History and Society, 1(2), 111-139.

Worm, A. (2019): Fluchtmigration aus Syrien. Eine biographietheoretische und figurationssoziologische Studie. Göttinger Beiträge zur soziologischen Biographieforschung, Bd. 1. Göttingen: Universitätsverlag Göttingen. Online: https://doi.org/10.17875/gup2019-1228<09.04.2020>. 